\title{
Simultaneous Improvement of Lincomycin a Production and Reduction of Lincomycin B Levels in Streptomyces Lincolnensis Using a Combined Medium Optimization Approach
}

\section{Xiu-hong Zheng}

East China University of Science and Technology https://orcid.org/0000-0002-5589-4115

Rui-fang Ye ( $\sim$ rfye@ecust.edu.cn )

East China University of Science and Technology

Qi-hang Ding

East-China Institute of Technology: East China University of Science and Technology

Feng-xian Hu

East-China Institute of Technology: East China University of Science and Technology

Hong-zhou Zhang

Tofond

Shen Lai

Tofond

\section{Research Article}

Keywords: lincomycin, steepest ascent method, PCR analysis, fermentation, osmotic stress

Posted Date: September 20th, 2021

DOI: https://doi.org/10.21203/rs.3.rs-780307/v1

License: (c) (i) This work is licensed under a Creative Commons Attribution 4.0 International License.

Read Full License

Version of Record: A version of this preprint was published at Annals of Microbiology on April 4th, 2022. See the published version at https://doi.org/10.1186/s13213-022-01672-w. 


\section{Abstract}

\section{Purpose}

The current study aimed to optimize the culture and production parameters of industrial production of lincomycin A by Streptomyces lincolnensis using a statistical approach that could also reduce unwanted by-products.

\section{Methods}

The Plackett-Burman design, steepest ascent method, and response surface design were used to evaluate different factors that affect lincomycin A production.

Results

Using an optimized $S$. lincolnensis fermentation medium, lincomycin A production was increased up to $4600 \mathrm{mg} / \mathrm{L}$ in shaking flasks, which indicated a $28.3 \%$ improvement over previous production in an unoptimized medium ( $3585 \mathrm{mg} / \mathrm{L}$ ). Additionally, the concentration of lincomycin B by-product was reduced to $0.8 \%$, which was $82.2 \%$ lower than that in the un-optimized medium. Further, quantitative real-time PCR analysis revealed the optimized medium improved lincomycin A production by stimulating key genes in the lincomycin A biosynthesis pathway, as well as an osmotic stress gene.

\section{Conclusions}

Optimizing the fermentation medium improved lincomycin A production and decreased that of the lincomycin B by-product, which could help cut production costs and simplify downstream separation processes. We found that osmotic stress reduced the concentration of lincomycin B, which could also help reduce fermentation by-product yields in other actinobacteria.

\section{Background}

Streptomyces species produce a wide range of biologically active secondary metabolites, including antitumor agents, immunosuppressants, and antibiotics with clinical, agricultural, and veterinary uses (Bibb 2005). Among these products, lincomycin is a natural antimicrobial that belongs to the lincosamides class of antibiotics, which was first discovered in Streptomyces lincolnensis from Lincoln, Nebraska (Chang and Weisblum 1967; Hornish et al. 1987). Lincomycin is one of the most widely used antibiotics administered to animals and humans against Gram-positive bacteria; it exerts its antibacterial action by inhibiting protein synthesis and blocking the peptidyl transferase reaction on the 50S ribosome subunit in sensitive bacteria (Brahme et al. 1984; Řezanka and Spížek 2004). Recent research has focused on maximizing lincomycin production using $S$. lincolnensis by establishing fermentation conditions for commercial applications (Lee et al. 2014). The world market for lincomycin is at the scale of hundreds of tons per year. According to the ESVAC report, sales of lincosamides were approximately 
300 tons in the EU, which represented approximately $5 \%$ of the total sales of veterinary pharmaceuticals in 2017 (EMA/294674/2019).

In the industrial fermentation of $S$. lincolnensis, desirable lincomycin A, a 3C 4-alkyl-L-proline derivative (APD), is the main product. However, lincomycin B, which has a $2 C$ instead of a $3 C$ APD moiety, is an undesirable by-product that often appears as a minority component in production cultures (Argoudelis et al. 1964; Pang et al. 2015; Janata et al. 2018). It exhibits only $25 \%$ of the antibiotic activity as compared with lincomycin $A$ and has a high toxicity. According to the guidelines from Pharmacopoeias in the USA and China, lincomycin B content in a lincomycin formulation should be less than $5 \%$ (Pang et al. 2015). During fermentation, undesirable lincomycin B usually accounts for $7-10 \%$ of the total lincomycin content and must be removed by complicated downstream purification processes before it can be used as a marketable medicine or active pharmaceutical ingredient. Thus, it is necessary to maximize lincomycin A and minimize lincomycin B production through process optimization during fermentation.

To date, few studies have focused on reducing the production of lincomycin B in the upstream steps of fermentation. At present, reduction of lincomycin B production in the fermentation broth has mainly been through genetic engineering. The lincomycin $B$ content was reduced to $4.41 \%$ by overexpressing both ImbW and metK genes, which regulate APD and propylproline biosynthesis of lincomycin(Pang et al. 2015). The overexpression of $I m b B 1$ (structural gene) alone only reduced the content of lincomycin $B$ to 2.5\% (Yang et al. 2020). However, few studies have been conducted on reduction of lincomycin B content in the fermentation broth through fermentation engineering.

Production of secondary metabolites depends on the strain and species of microorganisms used as well as on the fermentation medium, which can significantly affect product yield. The traditional "one-factor at a time" optimization strategy requires a considerable amount of time and labor, and often leads to unreliable results and inaccurate conclusions. As an alternative, statistical strategy is an efficient approach to accounting for a large number of variables and has been effectively applied to optimize cultivation processes (Mansouriieh et al. 2019; Fridous et al. 2020; Eskandari and Etemadifar 2021).

For example, Plackett-Burman design (PBD), the steepest ascent method, and Box-Behnken Design (BBD) have all been successfully applied for determining the components or variables that have significant effects on the production of primary or secondary metabolites and have statistically optimized metabolic production (Di et al. 2017; Wang et al. 2019). By optimizing media components, the yield of lincomycin A was increased from $1 \mathrm{~g} / \mathrm{L}$ to $3.5 \mathrm{~g} / \mathrm{L}$ (Luthra et al. 2019). Moreover, adding phosphorus improved the fermentation titer of lincomycin A (Li et al. 2007). However, few papers have addressed the use of a design method for medium formulation to improve lincomycin A productivity while minimizing lincomycin B accumulation.

This study describes the successful optimization of a culture medium to produce lincomycin A with minimal lincomycin B production by an industrial lincomycin-producing strain of $S$. lincolnensis using statistical mixture designs. This was achieved by implementing PBD, steepest ascent, and BBD methodologies. Finally, we used transcription profiling to identify genes involved in the lincomycin 
biosynthetic pathway and osmotic pressure to improve our understanding of the mechanism underlying lincomycin accumulation in S. lincolnensis.

\section{Materials And Methods}

\section{Microorganism and culture conditions}

The strain S. lincolnensis 24 was kindly provided by Topfond Pharmaceutical Co., Henan, China. S. lincolnensis 24 strain was activated on slant medium for approximately 7 days at $30{ }^{\circ} \mathrm{C}$. Subsequently, approximately $0.5 \mathrm{~m}^{3}$ strains from the solid medium were inoculated into $25 \mathrm{~mL}$ of seed medium for $48 \mathrm{~h}$ in Erlenmeyer flasks $(250 \mathrm{~mL})$ at $30^{\circ} \mathrm{C}$ and at $220 \mathrm{rpm}$ as the first-grade seeds. Then, $10 \%$ of first-grade seed was transferred into $25 \mathrm{~mL}$ fermentation medium at $30^{\circ} \mathrm{C}$ and at $220 \mathrm{rpm}$ for 7 days.

The media used in this study included (1) slant medium: $19 \mathrm{~g} / \mathrm{L}$ soluble starch, $5 \mathrm{~g} / \mathrm{L}$ soybean powder, $0.5 \mathrm{~g} / \mathrm{L} \mathrm{NaCl}, 1 \mathrm{~g} / \mathrm{L} \mathrm{KNO}_{3}, 0.5 \mathrm{~g} / \mathrm{L} \mathrm{K}_{2} \mathrm{HPO}_{4}, 0.5 \mathrm{~g} / \mathrm{L} \mathrm{MgSO}_{4}, 0.01 \mathrm{~g} / \mathrm{L} \mathrm{FeSO}_{4}, 12 \mathrm{~g} / \mathrm{L}$ agar. (2) seed medium: $20 \mathrm{~g} / \mathrm{L}$ soybean powder, $10 \mathrm{~g} / \mathrm{L}$ glucose, $20 \mathrm{~g} / \mathrm{L}$ corn starch, $30 \mathrm{~g} / \mathrm{L}$ corn steep liquor, $2 \mathrm{~g} / \mathrm{L}$ $\left(\mathrm{NH}_{4}\right)_{2} \mathrm{SO}_{4}$ and $5 \mathrm{~g} / \mathrm{L} \mathrm{CaCO}_{3}$; (3) initial fermentation medium (control): $25 \mathrm{~g} / \mathrm{L}$ soybean powder, $2 \mathrm{~g} / \mathrm{L}$ corn steep liquor, $5 \mathrm{~g} / \mathrm{L} \mathrm{NaCl}, 8 \mathrm{~g} / \mathrm{L} \mathrm{NaNO}_{3}, 0.2 \mathrm{~g} / \mathrm{L} \mathrm{KH}_{2} \mathrm{PO}_{4}, 8 \mathrm{~g} / \mathrm{L}\left(\mathrm{NH}_{4}\right)_{2} \mathrm{SO}_{4}, 100 \mathrm{~g} / \mathrm{L}$ glucose, $8 \mathrm{~g} / \mathrm{L}$ $\mathrm{CaCO}_{3}$. (4) optimize fermentation medium (OP): Except for corn steep liquor (1.4 g/L) and glucose (126 $\mathrm{g} / \mathrm{L})$, other components were maintained constant as initial fermentation medium (4) Medium-1 (M-1): Except for $\mathrm{NaCl}(10 \mathrm{~g} / \mathrm{L})$, other components were maintained constant as initial fermentation medium. (5) Medium-2 (M-2): Except for glucose (126 g/L), other components were maintained constant as initial fermentation medium. (6) Medium-3 (M-3): Except for corn steep liquor (1.4 g/L), other components were maintained constant as initial fermentation medium.

\section{Analytical Method}

Lincomycin A and lincomycin B were quantified by high-performance liquid chromatography (HPLC) (Agilent Technologies, Santa Clara, CA) on Eclipse XDB-C18 column $(5 \mu \mathrm{m}$, i.d. $4.6 \times 250 \mathrm{~mm})$. The samples were eluted with $60 \%(\mathrm{~V} / \mathrm{v})$ methanol and $40 \%(\mathrm{~V} / \mathrm{v}) 50 \mathrm{mM}$ ammonium acetate at a flow rate of $0.6 \mathrm{~mL} / \mathrm{min}\left(30^{\circ} \mathrm{C}\right)$. The lincomycin peak was separated and detected by UV absorbance at $210 \mathrm{~nm}$. The peak areas were proportional to the concentration of lincomycin. The injection volume was $20 \mu \mathrm{L}$, and the retention time of lincomycin was about $15 \mathrm{~min}$. The supernatant of the fermentation broth is chemically analyzed for reducing sugar level via the Fehling reagent (Xu et al. 2004).

The packed mycelia volume (PMV) value was the percentage of precipitate volume to broth volume (Zhang et al. 2019).

Supernatant samples were obtained by centrifugation at 3000rpm for $10 \mathrm{~min}$. The $600 \mu \mathrm{L}$ centrifuged supernatant of a lincomycin culture or broth was transferred to EP tube. Then the osmotic pressure of the fermentation broth was determined with an automatic freezing point osmometer FM-8P (Shanghai, China) (Wucherpfennig et al. 2011; Song et al. 2018). 


\section{Quantitative RTPCR analysis}

The sample time for qRT-PCR was set at $48 \mathrm{~h}$. Spin Column Bacteria Total RNA Purification Kit (Sanhon Biotech, China) was used for the total RNA extraction and HiFiScript gDNA Removal RT MasterMix (Kangwei Century Biotechnology Co., Ltd., Taizhou, China) for CDNA synthesis, following procedures recommended by the manufacturers. The reverse transcription procedure was as follow: $37^{\circ} \mathrm{C}$ for $15 \mathrm{~min}$, $85^{\circ} \mathrm{C}$ for $5 \mathrm{~s}$. Quantitative Real-time PCR was performed using the Bio-Rad CFX96 Real-Time PCR detection system (Bio-Rad Laboratories, Inc., Richmond, CA). Ultra SYBR Mixture (Kangwei Century Biotechnology Co., Ltd.) was used to prepare the reaction solution at low temperature, according to the instructions. PCR was performed using the following protocol: $95^{\circ} \mathrm{C}$ for 10 minutes, 40 cycles of $95^{\circ} \mathrm{C}$ for $30 \mathrm{~s}, 60^{\circ} \mathrm{C}$ for $1 \mathrm{~min}$. qRT-PCR was conducted in triplicate for each sample. With the transcription level of each gene in the control group as the reference unit, relative gene quantification was originally performed using the comparison $2^{-\triangle \Delta C T}$ method (Livak and Schmittgen 2001) and normalized to $16 S$ rDNA.

\section{Plackett-Burman design}

The Plackett-Burman design was used to screen the most important medium components for lincomycin A production. In general, this design is a two-factorial design, which identifies the significant influencing factors required for improved targeted production by screening $n$ variables in $n+1$ experiments (Burman and Plackett 1946). In this experiment, eight independent and three dummy variables were selected for the screening in 12 trials. Each variable was represented at two levels high and low (Table 1). The variables chosen for the present study were soybean powder $\left(X_{1}\right)$, corn steep liquor $\left(X_{2}\right), \mathrm{NaCl}\left(X_{3}\right), \mathrm{NaNO}_{3}$ $\left(X_{4}\right), \mathrm{KH}_{2} \mathrm{PO}_{4}\left(\mathrm{X}_{5}\right),\left(\mathrm{NH}_{4}\right)_{2} \mathrm{SO}_{4}\left(\mathrm{X}_{6}\right)$, glucose $\left(\mathrm{X}_{7}\right)$ and $\mathrm{CaCO}_{3}\left(\mathrm{X}_{8}\right)$. 
Table 1

Three-level design of Plackett-Burman

experiment

\begin{tabular}{|llll|}
\hline Source & \multicolumn{3}{l}{ Levels $(\mathrm{g} / \mathrm{L})$} \\
\cline { 2 - 5 } & -1 & $\mathbf{0}$ & $\mathbf{+ 1}$ \\
\hline $\mathrm{X}_{1}$ soybean powder & 20 & 25 & 30 \\
\hline $\mathrm{X}_{2}$ corn steep liquor & 1.6 & 2 & 2.4 \\
\hline $\mathrm{X}_{3} \mathrm{NaCl}$ & 4 & 5 & 6 \\
\hline $\mathrm{X}_{4} \mathrm{NaNO}_{3}$ & 6.4 & 8 & 9.6 \\
\hline $\mathrm{X}_{5} \mathrm{KH}_{2} \mathrm{PO}_{4}$ & 0.16 & 0.20 & 0.24 \\
\hline $\mathrm{X}_{6}\left(\mathrm{NH}_{4}\right)_{2} \mathrm{SO}_{4}$ & 6.4 & 8 & 9.6 \\
\hline $\mathrm{X}_{7} \mathrm{glucose}$ & 80 & 100 & 120 \\
\hline $\mathrm{X}_{8} \mathrm{CaCO}_{3}$ & 6.4 & 8 & 9.6 \\
\hline
\end{tabular}

\section{Statistical experimental designs}

During medium optimization, response surface methodology (RSM) can assess the relationships between the independent variables and the responses with useful mathematical and statistical techniques. The two most common designs used in RSM are the central composite design (CCD) and Box-Behnken design (BBD). Compared to the CCD method, the BBD is considered the most suitable for evaluating quadratic response surfaces, and is much easier to realize because it requires less factor levels, (Dranca et al. 2020). BBD is a rotatable three-level design, which is used for the investigation of response surface method and the constructing a second-order polynomial model. The respective levels for the three variables are shown in (Table S1), and the concentrations of the other variables were set at zero levels. The results were described using the following equation:

$Y=\beta_{0}+\beta_{1} X_{1}+\beta_{2} X_{2}+\beta_{3} X_{7}+\beta_{11} X_{2}^{2}+\beta_{22} X_{2}^{2}+\beta_{33} X_{7}^{2}+\beta_{12} X_{1} X_{2}+\beta_{13} X_{1} X_{7}+\beta_{23} X_{2} X_{7}(1)$,

where $Y$ represents the dependent variable (lincomycin A or lincomycin $B$ content), $\beta_{0}$ represents the model constant, $\beta_{1}, \beta_{2}$ and $\beta_{3}$ represent the linear coefficients, $\beta_{11}, \beta_{22}$ and $\beta_{33}$ represent the squared coefficients, and $\beta_{12}, \beta_{13}$ and $\beta_{23}$ represent interaction coefficients (Chen et al. 2020).

\section{Results And Discussion}

Plackett-Burman design to improve lincomycin A production in S. lincolnensis fermentation 
PBD was developed using Design-Expert software (Wang et al. 2019). The design matrix selected for the screening of significant variables for lincomycin A and lincomycin B production (Table 2). The confidence level was set at $5 \%$; therefore, the variables that scored a probability $(p)$ value less than 0.05 were considered significantly influential factors that could affect lincomycin production. The statistical analysis of the model was used an analysis of variance (ANOVA) to evaluate the significance and effectiveness of the design. The ANOVA for lincomycin A content showed that soybean powder, corn steep liquor, glucose, and $\mathrm{CaCO}_{3}$ were components of the culture medium that significantly affected lincomycin A production, as their $p$-values were less than 0.05 (Table S2). ANOVA analysis of lincomycin B content showed that soybean powder, corn steep liquor, glucose, and $\mathrm{NaNO}_{3}$ were components that had a significant effect on lincomycin A production, as their p-values were also less than 0.05 (Table S2). Lincomycin A and lincomycin B content was significantly affected in media that contained soybean powder, corn steep liquor, and glucose. Thus, the important factors identified by the initial PlackettBurman screening method, which influenced lincomycin A and lincomycin B content, were soybean powder, corn steep liquor, and glucose.

Table 2

Results of Plackett-Burman experiment

\begin{tabular}{|lcccccccccc|}
\hline Run & $\mathbf{X}_{\mathbf{1}}$ & $\mathbf{X}_{\mathbf{2}}$ & $\mathbf{X}_{\mathbf{3}}$ & $\mathbf{X}_{\mathbf{4}}$ & $\mathbf{X}_{\mathbf{5}}$ & $\mathbf{X}_{\mathbf{6}}$ & $\mathbf{X}_{\mathbf{7}}$ & $\mathbf{X}_{\mathbf{8}}$ & Lincomycin A (mg/L) & Lincomycin B (\%) \\
\hline 0 & 0 & 0 & 0 & 0 & 0 & 0 & 0 & 0 & 3555 & 4.23 \\
\hline 1 & +1 & -1 & +1 & +1 & -1 & -1 & -1 & +1 & 2884 & 1.54 \\
2 & -1 & +1 & +1 & +1 & +1 & +1 & -1 & -1 & 2625 & 1.89 \\
\hline 3 & -1 & -1 & -1 & -1 & -1 & -1 & -1 & -1 & 3706 & 5.43 \\
\hline 4 & +1 & -1 & -1 & +1 & +1 & +1 & +1 & +1 & 3937 & 4.36 \\
\hline 5 & +1 & +1 & +1 & +1 & -1 & -1 & +1 & -1 & 3083 & 1.08 \\
\hline 6 & -1 & +1 & -1 & +1 & +1 & -1 & -1 & +1 & 3124 & 2.15 \\
\hline 7 & +1 & +1 & -1 & -1 & -1 & +1 & -1 & +1 & 1837 & 2.62 \\
\hline 8 & +1 & -1 & +1 & -1 & +1 & +1 & -1 & -1 & 1568 & 4.69 \\
\hline 9 & -1 & -1 & -1 & -1 & -1 & +1 & +1 & -1 & 4364 & 4.38 \\
\hline 10 & +1 & +1 & -1 & -1 & +1 & -1 & +1 & -1 & 2624 & 4.83 \\
\hline 11 & -1 & -1 & +1 & -1 & +1 & -1 & +1 & +1 & 4542 & 4.62 \\
\hline 12 & -1 & +1 & +1 & -1 & -1 & +1 & +1 & +1 & 4439 & \\
\hline The values were obtained from three independent experiments. & \\
\hline
\end{tabular}




\section{Steepest ascent improved culture conditions for optimizing lincomycin A production}

Because soybean powder and corn steep liquor exerted negative effects on lincomycin A and glucose exerted a positive effect (Table S2), the direction of steepest ascent indicated that the concentration of glucose should increase and the concentrations of soybean powder and corn steep liquor should decrease for optimal experimental conditions that maximize lincomycin A production. Five sets of experiments using the steepest ascent and corresponding experimental results showed that the optimum value area was located in group Run3 (Table 3).

Table 3

Three-factors steepest ascent experiment

\begin{tabular}{|llllll|}
\hline Run & $\mathbf{X}_{1}(\mathbf{g} / \mathbf{L})$ & $\mathbf{X}_{2}(\mathbf{g} / \mathbf{L})$ & $\mathbf{X}_{\mathbf{7}}(\mathbf{g} / \mathrm{L})$ & Lincomycin A (mg/L) & Lincomycin B (\%) \\
\hline 0 & 25 & 2 & 100 & $3536 \pm 82$ & $4.4 \pm 0.2$ \\
\hline 1 & 15 & 1.2 & 140 & $3421 \pm 102$ & $5.3 \pm 0.2$ \\
\hline 2 & 20 & 1.3 & 133 & $3875 \pm 48$ & $2.8 \pm 0.1$ \\
\hline 3 & 25 & 1.4 & 126 & $4606 \pm 101$ & $0.8 \pm 0.1$ \\
\hline 4 & 30 & 1.5 & 119 & $3671 \pm 152$ & $2.4 \pm 0.1$ \\
\hline 5 & 35 & 1.6 & 112 & $3415 \pm 72$ & $3.7 \pm 0.2$ \\
\hline 6 & 40 & 1.7 & 105 & $2299 \pm 96$ & $3.6 \pm 0.1$ \\
\hline 7 & 45 & 1.8 & 98 & $1029 \pm 22$ & $7.2 \pm 0.3$ \\
\hline
\end{tabular}

These results helped define the optimal concentrations as $25 \mathrm{~g} / \mathrm{L}$ soybean powder, $1.4 \mathrm{~g} / \mathrm{L}$ corn steep liquor, and $126 \mathrm{~g} / \mathrm{L}$ glucose; further suggesting that lincomycin $A$ and lincomycin B were proximal to the region of maximum production. Accordingly, the concentrations of the three medium ingredients in the the third out of five experiments were considered the center point of BBD.

\section{Box-Behnken design experimental optimization and response surface analysis of growth conditions that affect lincomycin production}

BBD is a well-known optimization method based on the establishment of a mathematical model that assesses the statistical significance of the effects of different factors on the final response (Annadurai and Sheeja 1998). The BBD method was used to assess the effect of soybean powder, corn steep liquor, glucose concentrations on lincomycin $A$ and $B$ production. A total of 17 experiments with different combinations of nutrient concentrations were performed and their effects on lincomycin $A$ and $B$ 
production are presented in Table 4. Multiple regression analysis of the experimental data used a secondorder polynomial equation derived for the lincomycin A yield and lincomycin B content using the significant terms:

Table 4

Box-Behnken Design experimental design

\begin{tabular}{|llllll|}
\hline Run & $\mathbf{X}_{\mathbf{1}}(\mathbf{g} / \mathrm{L})$ & $\mathbf{X}_{2}(\mathbf{g} / \mathrm{L})$ & $\mathbf{X}_{\mathbf{7}}(\mathbf{g} / \mathrm{L})$ & Lincomycin A (mg/L) & Lincomycin B (\%) \\
\hline 0 & 25 & 2 & 100 & $3519 \pm 71$ & $4.8 \pm 0.2$ \\
\hline 1 & 25 & 1.68 & 151.2 & $4078 \pm 39$ & $3.0 \pm 0.1$ \\
\hline 2 & 20 & 1.40 & 151.2 & $3464 \pm 64$ & $3.6 \pm 0.1$ \\
\hline 3 & 25 & 1.68 & 100.8 & $4511 \pm 36$ & $3.5 \pm 0.2$ \\
\hline 4 & 30 & 1.40 & 100.8 & $3752 \pm 75$ & $4.3 \pm 0.2$ \\
\hline 5 & 20 & 1.12 & 126 & $3712 \pm 61$ & $2.8 \pm 0.2$ \\
\hline 6 & 30 & 1.68 & 126 & $3452 \pm 54$ & $2.9 \pm 0.1$ \\
\hline 7 & 20 & 1.68 & 126 & $4375 \pm 122$ & $2.6 \pm 0.2$ \\
\hline 8 & 30 & 1.40 & 151.2 & $2558 \pm 102$ & $4.3 \pm 0.2$ \\
\hline 9 & 25 & 1.12 & 100.8 & $4711 \pm 58$ & $3.9 \pm 0.1$ \\
\hline 10 & 30 & 1.12 & 126 & $3734 \pm 193$ & $2.5 \pm 0.2$ \\
\hline 11 & 25 & 1.12 & 151.2 & $3820 \pm 19$ & $2.7 \pm 0.1$ \\
\hline 12 & 20 & 1.40 & 100.8 & $3712 \pm 15$ & $3.4 \pm 0.1$ \\
\hline 13 & 25 & 1.40 & 126 & $4500 \pm 41$ & $1.0 \pm 0.1$ \\
\hline 14 & 25 & 1.40 & 126 & $4580 \pm 46$ & $0.8 \pm 0.1$ \\
\hline 15 & 25 & 1.40 & 126 & $4608 \pm 78$ & $1.3 \pm 0.1$ \\
\hline 16 & 25 & 1.40 & 126 & $4612 \pm 43$ & $0.8 \pm 0.1$ \\
\hline 17 & 25 & 1.40 & 126 & $4548 \pm 57$ & \\
\hline The values were obtained from three independent experiments. & \\
\hline & & & & & \\
\hline
\end{tabular}

$Y_{1}=4569.6-220.88 \times X_{1}+54.87 \times X_{2}-345.75 \times X_{7}-236.25 \times X_{1}-236.50 \times X_{1} X_{7}+114.50 \times X_{2} X_{7}-829.93 \times X_{1} 2+$ $78.57 \times X_{2} 2-368.17 \times X_{7} 2(2)$

$\mathrm{Y}_{2}=0.96+0.20 \times \mathrm{X}_{1}+0.013 \times \mathrm{X}_{2}-0.19 \times \mathrm{X}_{7}+0.15 \times \mathrm{X}_{1} \mathrm{X}_{2}-0.05 \times 10^{-3} \times \mathrm{X}_{1} \mathrm{X}_{7}+0.17 \times \mathrm{X}_{2} \mathrm{X}_{7}+1.18 \times \mathrm{X}_{1} 2+0.56 \times \mathrm{X}_{2} 2$ $+1.76 \times \mathrm{X}_{7} 2(3)$ 
where $Y_{1}(\mathrm{mg} / \mathrm{L})$ is the predicted lincomycin $A$ yield; $Y_{2}(\%)$ is the predicted lincomycin $B$ content; and $X_{1}$, $X_{2}$, and $X_{7}$ are the coded values of soybean powder, corn steep liquor, and glucose, respectively.

Statistical significance of the response surface model and all the coefficient estimates were assessed with ANOVA (Tables S3 and S4). The high F-value (180.64) and a very low p-value $(<0.0001)$ suggested that the model was highly significant, while an insignificant lack of fit $(p=0.2201>0.05)$ revealed the effectiveness of the regression analysis, suggesting that the regression model could fit the effect of the three culture factors on lincomycin A content. The ratio of the explained and total variation indicated that the coefficient of determination $\left(R^{2}\right)$ could be used to assess the goodness of the model. The value of $R^{2}$ was $99.57 \%$, which indicated that only $0.43 \%$ of the lincomycin A content variability could not be explained by the predicted equation of the model. The Adj- $R^{2}$ value of $99.02 \%$ further validated the significance of this model. A low coefficient of variation (CV) $(C V=1.44 \%)$ value revealed that the deviations between the predicted and experimental values were low, and it displayed a high degree of precision and reliability in the conducted experiments. "Adeq Precision" provides the signal-to-noise ratio and a ratio greater than four is desirable. In this study, a ratio (47.512) greater than 4 indicated the use of this model in future studies will be supported.

The ANOVA showed that the model for lincomycin B content had a $p$-value $=0.0006$, suggested that the model was highly significant (Table S3). The lack of fit $(p=0.1252>0.05)$ was insignificant, suggesting that the regression model could fit the effect of the three culture factors on lincomycin $B$ content.

The $R^{2}$ was 0.9628 , Adj- $R^{2}$ was 0.9149 , and the ratio was 14.541 ; these results indicated a high degree of precision and high reliability for this model, which supported its use in future studies. The content of lincomycin A and lincomycin B changed with changes in soybean powder, corn steep liquor and glucose concentrations, and their corresponding $3 \mathrm{D}$ response surfaces were generated to better determine the interaction of variables with the corresponding variables (Fig. 1). The maximum lincomycin $A$ and lincomycin B contents were $4569.6 \mathrm{mg} / \mathrm{L}$ and $0.96 \%$, respectively, with $25 \mathrm{~g} / \mathrm{L}$ soybean powder $\left(X_{1}\right), 1.40$ $\mathrm{g} / \mathrm{L}$ corn steep liquor $\left(X_{2}\right)$, and $126 \mathrm{~g} / \mathrm{L}$ glucose $\left(X_{7}\right)$.

Fermentation was conducted and lincomycin production in broth was evaluated by HPLC (Fig. 2). The results for the validation experiment showed that the values for the two responses of lincomycin $A$ and lincomycin B contents were in close agreement with the predicted values. When $25 \mathrm{~g} / \mathrm{L}$ soybean powder, $1.40 \mathrm{~g} / \mathrm{L}$ corn steep liquor, and $126 \mathrm{~g} / \mathrm{L}$ glucose were used, the content of lincomycin A increased from $3585 \pm 110 \mathrm{mg} / \mathrm{L}$ to $4600 \pm 134 \mathrm{mg} / \mathrm{L}$, and that of lincomycin $B$ decreased from $4.5 \% \pm 0.2 \%$ to $0.8 \pm 0.1 \%$. These concentrations verified the accuracy of the statistical model.

\section{Assay of lincomycin A, lincomycin B, and fermentation parameters}

Glucose is not only a carbon source but can also affect the osmotic stress of a culture medium at certain concentrations, while corn steep liquor provides various amino acids, vitamins, and metal ions, etc. To explore possible causes of the increased lincomycin A and decreased lincomycin B after medium 
optimization, the fermentation parameters for S. lincolnensis 24 were measured (Fig. 3) using samples collected every $24 \mathrm{~h}$.

The optimized medium not only influenced the nutrient content of the culture, such as sugar (Fig. 3A), it also affected the growth environment, such as osmotic stress and $\mathrm{pH}$ parameters (Fig. 3A and B). As shown in Fig. $3 \mathrm{~A}$ and $3 \mathrm{~B}$, reducing the sugar and osmotic stress in the optimized medium were always higher than the initial medium before $144 \mathrm{~h}$. At the end of $168 \mathrm{~h}$ of fermentation, the reducing sugars in and osmotic stress of the optimized medium were the same as the initial medium, with the reducing sugar consumed completely. These results indicated that changes in glucose affected the reducing sugar and osmotic stress of the medium. We hypothesized that these variations in the environment and nutrients affected the primary metabolism of S. lincolnensis and caused PMV changes (Fig. 3C), as well as changes in the secondary metabolism of $S$. lincolnensis, which improved lincomycin A quantity and quality (Fig. 3D). As shown in Fig. 3D, the optimized medium produced lincomycin A before $48 \mathrm{~h}$, while the initial medium produced lincomycin $A$ after $48 \mathrm{~h}$. For lincomycin $B$, only the initial medium produced lincomycin B before $48 \mathrm{~h}$. For both the initial and optimized media, both quickly increased lincomycin $\mathrm{A}$ and decreased lincomycin B in the 72-96 h stage.

\section{Influence of corn steep liquor, glucose, and osmotic stress on the optimized medium conditions}

In the present study, the increased glucose concentration affected the osmotic stress environment of the fermentation, which benefited S. lincolnensis growth and the reduction of lincomycin B accumulation (Fig. 3). We hypothesized that the change in osmotic stress had a significant influence on cell growth and lincomycin synthesis. Therefore, to explore the role of osmotic stress in lincomycin fermentation, $\mathrm{NaCl}$ was used to regulate osmotic stress in the initial medium. To determine the effect of salt-stress sensitivity on lincomycin production, $S$. lincolnensis was grown in the initial medium (control, containing $5 \mathrm{~g} / \mathrm{L}$ $\mathrm{NaCl}$ ), optimized medium (OP, initial medium with $1.4 \mathrm{~g} / \mathrm{L}$ corn steep liquor and $126 \mathrm{~g} / \mathrm{L}$ glucose), initial medium with $10 \mathrm{~g} / \mathrm{L} \mathrm{NaCl}$ (M1), initial medium with $126 \mathrm{~g} / \mathrm{L}$ glucose (M2), or initial medium with $1.4 \mathrm{~g} / \mathrm{L}$ corn steep (M3). The osmotic pressure of the OP and M1-M2 media was similar. As shown in Fig. 4, the OP medium produced the highest lincomycin $A$ and lowest lincomycin B concentrations. Increasing the salt stress with $10 \mathrm{~g} / \mathrm{L} \mathrm{NaCl}$ in the initial medium (M1) resulted in a dramatic decrease in lincomycin $B$ production. Similarly, under glucose (M2) and corn steep liquor (M3) salt-stress conditions, a knockdown of about 31.8-63.6\% was achieved for lincomycin B production when compared with the control medium. These observations indicated that the osmotic conditions decreased lincomycin B concentrations during shake flask fermentation.

\section{Transcriptional analysis of lincomycin biosynthesis-related genes}

To further understand the observed variations between lincomycin production in initial and optimized media, quantitative real-time PCR (qRT-PCR) gene expression analysis was performed. In the biosynthesis of lincomycin, ImbW is an important methylase gene that is responsible for the C-methylation of 4-n- 
propyl-L-proline and determines whether 4- n-propyl-L-proline synthesizes lincomycin A or lincomycin B (Pang et al. 2015). The regulatory genes in lincomycin synthesis are divided into the lincomycin biosynthesis gene cluster (Imb cluster) and non-Imb genes, which are ImbU and SLCG_Lrp, respectively. These genes play positive regulatory roles in lincomycin biosynthesis (Hou et al. 2018; Xu et al. 2020). The ImbB1 gene encodes for a 2,3-extradiol cleavage enzyme that breaks down the L-3,4dihydroxyphenylalanine (L-DOPA) aromatic ring (Novotná et al. 2004). Replacing the tyrosinase involved in melanin synthesis, LmbB1 is assumed to be a dioxygenase that catalyzes the cleavage of 2,3-extradiol in the aromatic ring of L-3,4-dihydroxyphenyl alanine (Zhong et al. 2017). LmbB1 is a key gene that determines whether L-DOPA follows the lincomycin or the melanin biosynthesis pathway. Overexpression of ImbB1 can increase lincomycin A and decrease lincomycin B and melanin (Yang et al. 2020). These genes were selected based on their roles in the synthesis of lincomycin A and lincomycin B. Furthermore, osmotic stress in the optimized medium was higher than that of the initial medium (Fig. 3A); therefore, the transcript level of the osmotic stress related gene ( $m s c l$; GenBank: ANS65846.1) was also determined.

RNA was isolated from both initial and optimized media samples collected after $48 \mathrm{~h}$ of fermentation. qRT-PCR was conducted in triplicate for each sample, using 16S rRNA as an internal control (RT primers are listed in Table 5). The qRT-PCR data showed that ImbU, SLCG_Lrp, ImbW and ImbB1 expression was significantly higher in the optimized medium than in the initial medium (2-to 4-fold increase; Fig. 5). One study recently reported that $\mathrm{LmbU}$ is a significant pleiotropic transcriptional regulator in lincomycin biosynthesis that activates the $I m b$ cluster, including $I m b W$ and $I m b B 1$ in S. lincolnensis (Lin et al. 2020). Another study showed that SLCG_Lrp is a positive regulator for lincomycin biosynthesis through the direct induction of $I m b$ cluster genes, such as $I m b U$ (Xu et al. 2020). Our results indicated that increased lincomycin A production in the optimized medium was likely to be associated with the overexpression of the key genes involved in the lincomycin biosynthesis pathway, as well as some of the key regulators of the pathway. 
Table 5

Primer sequences

\begin{tabular}{|ll|}
\hline name & primer sequences $\mathbf{5}^{\prime} \rightarrow \mathbf{3}^{\prime}$ \\
\hline SLCG_Lrp & (F)-TCGTCGTACAGCCGCTGGTAG \\
& (R)-GATCGCGGAAGTGGTGGATGC \\
ImbU & (F)-GCGTAGTTGCGGATCGTCTGG \\
(R)-ACTCATCGGCCTGGTGTCTGG & (F)-CCTCATGGTCCTGCCGATGT \\
& (R)-AGCTCGCTCACCTCGATGAC \\
ImbW & (F)-A G C T G C T G G C C G A G G G C G T A \\
& (R)-G C C G C C G G A C T T G G A C G A C A \\
ImbB1 & (F)-AGTAAAGTCAATGCCGCCCGTATC \\
& (R)-GAATGTGTCGAGGGTCCAGGAAC \\
\hline $16 S$ rRNA & (F)-GCATCTGTGGTGGTTGAAAG \\
& (R)-CGTGTCTCAGTCCCAGTGTG \\
\hline
\end{tabular}

Microorganisms cope with environmental stress factors during industrial fermentation processes, such as osmotic, temperature, and oxidative stress stresses, which can significantly impact their primary and secondary metabolism (Lee et al. 2005; Li et al. 2009). Osmotic stress has an important effect on the secondary metabolism of Streptomyces. For example, disruption of either osaBSa, which encodes a response regulator protein, led to increased production of oligomycin up to $200 \%$, and avermectin up to $37 \%$ (Godinez et al. 2015). Regulation of the osmotic stress response can impact both development and antibiotic production in the model streptomycete, S. coelicolor (Bishop et al. 2004; Martínez et al. 2010). Bacteria have two families of mechanosensitive (MS) channels: small conductance (MscS) and large conductance (MscL) channels (Blount and Moe 2005; Blount and Iscla 2012). The majority of bacteria contain a single copy of $m s c L$, which is highly conserved between species (Wray et al. 2019). In bacteria, MS channels act as emergency release valves; when bacteria are exposed to high osmotic pressure, they will transport ( $\mathrm{K}^{+}$, glutamate, betaine, and proline) and synthesize (glutamate, trehalose, proline, and betaine) solutes to balance the increase in external osmotic pressure to maintain high cell turgor, which is a requisite for cell growth and division.

In this study, the optimized medium enhanced the osmotic stress of the fermentation condition and led to a 6.2 -fold increase in the transcription of $\mathrm{msc} /$ when compared with the initial medium (Fig. 5). The expression levels of $I \mathrm{mb} U$ were 3.9 -fold up-regulated in the optimized medium after $48 \mathrm{~h}$ compared to those of initial medium (Fig. 5). Recent studies have suggested LmbU can bind to the regions upstream of the $I m b A$ and $I m b W$ genes at the consensus and palindromic sequence 5'-CGCCGGCG-3' (Hou et al. 
2018). By sequence alignment, this palindromic sequence was located $184 \mathrm{bp}$ upstream of the $\mathrm{msc}$ gene (Fig. 6). LmbU binds directly to the regions upstream of $I \mathrm{mbW}$ and activates transcription. Thus, LmbU may also actively inhibit the production of lincomycin B (Hou et al. 2018; Pang et al. 2015). Higher osmotic stress might result in the down-regulation of lincomycin B biosynthesis (Fig. 4) due to LmbU targeting $\mathrm{mscl}$ and affecting the expression of $I m b W$ during lincomycin B biosynthesis.

As shown in Fig. 3D, under the optimized conditions, the emergence time of lincomycin A was enhanced, which effectively reduced the fermentation period. Previous studies showed that the pneumocandin $B_{0}$ fermentation period was significantly shorter when Glarea lozoyensis were cultured in a high osmolarity medium (Song et al. 2018); thus, osmotic stress could shorten the fermentation period in other bacteria. Moreover, simultaneously with the enhanced production time of lincomycin A, that of lincomycin B was delayed by approximately $24 \mathrm{~h}$ (Fig. 3D). These results suggest a temporal order and interconversion of lincomycin A and lincomycin B biosynthesis that are related to osmotic stress. The mechanism stated for lincomycin $A$ and lincomycin $B$ interconversion has not been reported. This study provides a foundation for interconversion of lincomycin A and lincomycin B in S. lincolnensis, although further research is needed to develop its application potential.

\section{Conclusion}

In the present study, we aimed to increase lincomycin A production, while simultaneously reducing lincomycin B level using a medium optimization approach. For this purpose, PBD, steepest ascent, and BBD methodologies were used to evaluate the influence of soybean powder, corn steep liquor, $\mathrm{NaCl}$, $\mathrm{NaNO}_{3}, \mathrm{KH}_{2} \mathrm{PO}_{4},\left(\mathrm{NH}_{4}\right)_{2} \mathrm{SO}_{4}$, glucose, and $\mathrm{CaCO}_{3}$, on lincomycin production using the industrial strain of S. lincolnensis. After $168 \mathrm{~h}$ of culture in an optimized medium, the lincomycin A titer was significantly increased, while the unwanted lincomycin B by-product decreased. The enhancement of lincomycin A production in the optimized medium could be attributed to the expression of the key genes in the lincomycin biosynthesis pathway as well as other regulator genes. Furthermore, the reduced lincomycin B accumulation could be related to increased osmotic stress, which should be the focus of future studies.

\section{Declarations}

\section{Ethics approval and consent to participate}

Not applicable.

\section{Consent for publication}

Not applicable.

\section{Availability of data and materials}


The data sets used and/or analyzed during the current study are available from the corresponding author on a reasonable request.

\section{Competing interests}

The authors declare that they have no competing interests.

\section{Funding}

This research had not received any financial assistance.

\section{Authors' contributions}

This research was conducted under the guidance of RFY and FXH. QHD was the assistant who assisted $\mathrm{XHZ}$ in completing the experiment. The industrial strains used in the experiment will be provided in the $\mathrm{HZZ}$ and SL.

\section{Acknowledgements}

Not applicable.

\section{References}

1. Annadurai G, Sheeja RY (1998) Use of Box-Behnken design of experiments for the adsorption of verofix red using biopolymer. Bioprocess Eng 18(6):463-466. https://doi.org/ $10.1007 /$ s004490050472

2. Argoudelis AD, Fox JA, Mason DJ, \& Eble TE (1964) New lincomycin-related antibiotics. J Am Chem Soc 86(22):5044-5045. https://doi.org/10.1021/ja01076a090

3. Bibb MJ (2005) Regulation of secondary metabolism in streptomycetes. Curr Opin Microbiol 8:208215. https://doi.org/10.1016/j.mib.2005.02.016

4. Bishop A, Fielding S, Dyson P, Herron P (2004) Systematic Insertional Mutagenesis of a Streptomycete Genome: A Link Between Osmoadaptation and Antibiotic Production. CSHL 14(5):893-900. https://doi.org/10.1101/gr.1710304

5. Blount P, Iscla I (2012) Sensing and Responding to Membrane Tension: The Bacterial MscL Channel as a Model System. Biophys J 103(2):169-174. https://doi.org/ 10.1016/j.bpj.2012.06.021

6. Blount P, Moe P (2005) Assessment of potential stimuli for mechano-dependent gating of MscL: effects of pressure, tension, and lipid headgroups. Biochemistry 44(36):12239-12244. https://doi.org/10.1021/bi0509649

7. Brahme NM, Gonzalez JE, Rolls JP (1984) Biosynthesis of the lincomycins. 1. Studies using stable isotopes on the biosynthesis of the propyl-and ethyl-L-hygric acid moieties of lincomycins A and B. J Am Chem Soc 106(25):7873-7878. https://doi.org/10.1021/ja00337a038 
8. Chang FN, Weisblum B (1967) The specificity of lincomycin Binding to ribosomes. Biochemistry 6:836-843. https://doi.org/10.1021/bi00855a025

9. Di G, Jiao Y, Wu J, Liu Z, \& Chen Q (2017) Optimization of eps production and characterization by a halophilic bacterium, kocuria rosea zjuqh from chaka salt lake with response surface methodology. Molecules 22(5):814. https://doi.org/ 10.3390/molecules22050814

10. Dranca F, Ursachi F, Oroian M (2020) Bee Bread: Physicochemical Characterization and Phenolic Content Extraction Optimization. Foods 9(10):1358.

11. Eskandari S, Etemadifar Z (2021) Biocompatibility and radioprotection by newly characterized melanin pigment and its production from Dietzia schimae NM3 in optimized whey medium by response surface methodology. Ann Microbiol 71(1):17.

12. European Medicines Agency (2019) Sales of Veterinary Antimicrobial Agents in 31 European Countries in 2017:(EMA/294674/2019).

13. Fridous S, lqbal S, Anwar S (2020) Optimization and modeling of glyphosate biodegradation by a novel Comamonas odontotermitis P2 through response surface methodology - ScienceDirect. Pedosphere 30( 5):618-627.

14. Godinez O, Dyson P, Sol RD, González JB, Mejía A (2015) Targeting the Osmotic Stress Response for Strain Improvement of an Industrial Producer of Secondary Metabolites. J Microbiol Biotechnol 25(11):1787-1795. https://doi.org/10.4014/jmb.1503.03042

15. Hornish RE, Gosline RE, Nappier JM (1987) Comparative metabolism of lincomycin in the swine, chicken, and rat. Drug Metab Rev 18 (2-3):177-214. https://doi.org/ 10.3109/03602538708998305

16. Hou BB, Lin YW, Wu HZ, Guo MJ, Petkovic H (2018) The novel transcriptional regulator ImbU promotes lincomycin biosynthesis through regulating expression of its target genes in Streptomyces lincolnensis. J Bacteriol 200(2):e00447. https://doi.org/ 10.1128/jb.00447-17

17. Janata J, Kamenik Z, Gazak R, Kadlcik S, \& Najmanova L (2018) Biosynthesis and incorporation of an alkylproline-derivative (apd) precursor into complex natural products. Nat Prod Rep 35(3). https://doi.org/10.1039/c7np00047b.

18. Lee EJ, Karoonuthaisiri N, Kim HS, Park JH, Cha CJ, Kao CM, Roe JH (2005) A master regulator sigma B governs osmotic and oxidative response as well as differentiation via a network of sigma factors in Streptomyces coelicolor. Mol Microbiol 57(5):1252-1264. https://doi.org/10.1111/j.13652958.2005.04761.x

19. Lee Y, Lee MJ, Choi YE, Chun GT, Jeong YS (2014) Optimization of cultivation medium and fermentation parameters for lincomycin production by Streptomyces lincolnensis. Biotechnol Bioproc E 19(6): 1014-1021.

20. Li L, Bai LQ, Zhou XF, Deng ZX (2009) Enhanced validamycin production and gene expression at elevated temperature in Streptomyces hygroscopicus subsp. jingangensis 5008. Sci Bull 54(07):1204-1209. https://doi.org/10.1007/s11434-009-0128-0

21. Li XB, Zhao GR, Zheng H, and Yuan YJ (2007) Improved industrial fermentation of lincomycin by phosphorus feeding. Process Biochem 42(4):662-668. https://doi.org/ 


\subsection{6/j.procbio.2006.12.007}

22. Lin CY, Pang AP, Zhang Y, Qiao J, Zhao GR (2020) Comparative transcriptomic analysis reveals the significant pleiotropic regulatory effects of LmbU on lincomycin biosynthesis. Microb Cell Fact 19(4). https://doi.org/10.1186/s12934-020-01298-0

23. Lina C, Yi W, Mao S, Li ZQ, Zhang SH (2020) Statistical optimization of a cellulase from Aspergillus glaucus CCHA for hydrolyzing corn and rice straw by RSM to enhance yield of reducing sugar. Biotechnol Lett 42(16):583-595. https://doi.org/ 10.1007/s10529-020-02804-5

24. Livak KJ, Schmittgen TD (2001) Analysis of Relative Gene Expression Data using Real-Time Quantitative PCR and the $2^{-\triangle \triangle C T}$ Method. Methods. 25(4):402-408.

25. Luthra U, Trivedi A, Singh N, Bagwe V, \& Lade M (2019) Studies to explore the impact of Carbon \& Nitrogen source for Lincomycin production at shake flask level using Streptomyces lincolnensis. IJERD 7(1):270-276.

26. Mansouriieh N, Sohrabi M R, Khosravi M (2019) Optimization of profenofos organophosphorus pesticide degradation by zero-valent bimetallic nanoparticles using response surface methodology. Arab J Chem 12(8):s 2524-2532.

27. Martínez LF, Bishop A, Parkes L, Sol RD Dyson P (2010) Osmoregulation in Streptomyces coelicolor. modulation of SigB activity by OsaC. Mol Microbiol 71(5):1250-1262.https://doi.org/10.1111/j.13652958.2009.06599.x

28. Novotná J, Honzátko A, Bednár P, Kopecký J, Janata J, Spížek J (2004) I-3,4-Dihydroxyphenyl alanine-extradiol cleavage is followed by intramolecular cyclization in lincomycin biosynthesis. Eur $\mathrm{J}$ Biochem 271(18):3678-3683. https://doi.org/ 10.1111/j.1432-1033.2004.04308.x

29. Pang AP, Du L, Lin CY, Qiao J, \& Zhao GR (2015) Co-overexpression of ImbWand metK led to increased lincomycin a production and decreased byproduct lincomycin B content in an industrial strain of streptomyces lincolnensis. J Appl Microbiol 119(4):1064-1074. https://doi.org/ 10.1111/jam.12919

30. Song P, Huang BQ, Zhang S, Zhang K, Yuan K, Ji XJ, Ren LJ, Wen JP, Huang H (2018) Novel osmotic stress control strategy for improved pneumocandin BO production in Glarea lozoyensis combined with a mechanistic analysis at the transcriptome level. Appl Microbiol Biotechnol 102:10729-10742

31. Spížek T, Řezanka (2004) Lincomycin, cultivation of producing strains and biosynthesis. Appl Microbiol Biotechnol 63(5):510-519. https://doi.org/ 10.1007/s00253-003-1431-3

32. Wang L, Chen Y, Jiang L, Zhou H, Tan Z, Du S (2019) Optimizing Three Kinds of Coagulants Using Plackett-Burman and Response Surface Methodology for Phosphorus Removal from Domestic Sewage. Pol J Environ Stud 28(2):877-885.

33. Wray R, Herrera N, Iscla I(2019) An agonist of the MscL channel affects multiple bacterial species and increases membrane permeability and potency of common antibiotics. Mol Microbiol 112(3):896-905. https://doi.org/ 10.1111/mmi.14325

34. Wucherpfennig T, Hestler T, Krull R (2011) Morphology engineering-osmolality and its effect on Aspergillus niger morphology and productivity. Microb Cell Factories 10(1):1 
35. Xu WL, Li YZ, Zhang QS, and Zhu HS (2004) A Selective Conversion of Sulfides to Sulfoxides in 3Fluorophenol. Syn Comm 34(2):231-237. https://doi.org/ 10.1081/SCC-120027258

36. Xu YR, Tang YQ, Nian W, Liu J, Cai XL, Liu RH, Bai LQ, Zhang LX, Zhang BC (2020) Transcriptional regulation of a leucine-responsive regulatory protein for directly controlling lincomycin biosynthesis in Streptomyces lincolnensis. Appl Microbiol Biotechnol 104(6):2575-2587.

37. Yang J, Ye RF, Zhang HZ, Liu Y (2020) Amplification of ImbB1 gene in streptomyces lincolnensis improves quantity and quality of lincomycin A fermentation. Prep Biochem Biotechnol 50(6):529537. https://doi.org/10.1080/10826068.2019.1710714

38. Zhang Q, Hang HF, Tian X, Zeng W, Yu Z, Zhuang YP, Chu J (2019) Combined available nitrogen resources enhanced erythromycin production and preliminary exploration of metabolic flux analysis under nitrogen perturbations. Bioprocess Biosyst Eng 42(11):1747-1756. https://doi.org/10.1007/s00449-019-02171-0

39. Zhong G, Zhao Q, Zhang Q, Liu W (2017) 4-alkyl-L-(Dehydro)proline biosynthesis in actinobacteria involves $\mathrm{N}$-terminal nucleophile-hydrolase activity of $\mathrm{Y}$-glutamyltranspeptidase homolog for $\mathrm{C}$ - $\mathrm{C}$ bond cleavage. Nat Commun 8:16109. https://doi.org/10.1038/ncomms16109

\section{Figures}



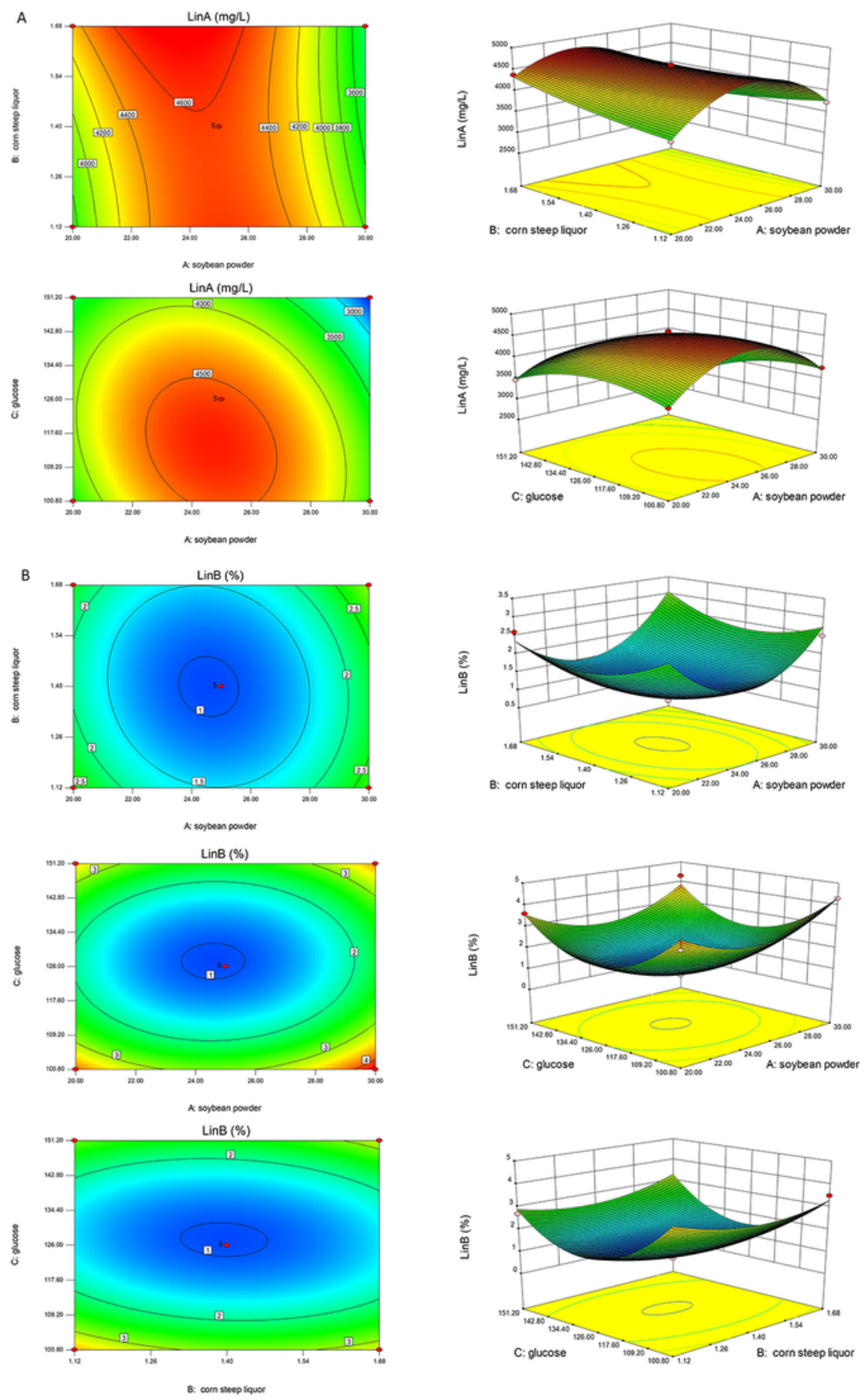

\section{Figure 1}

Contour (left) and response surface (right) plots of the effects of soybean powder, corn steep liquor, and glucose on lincomycin $A(A)$ and lincomycin $B(B)$ concentration. 


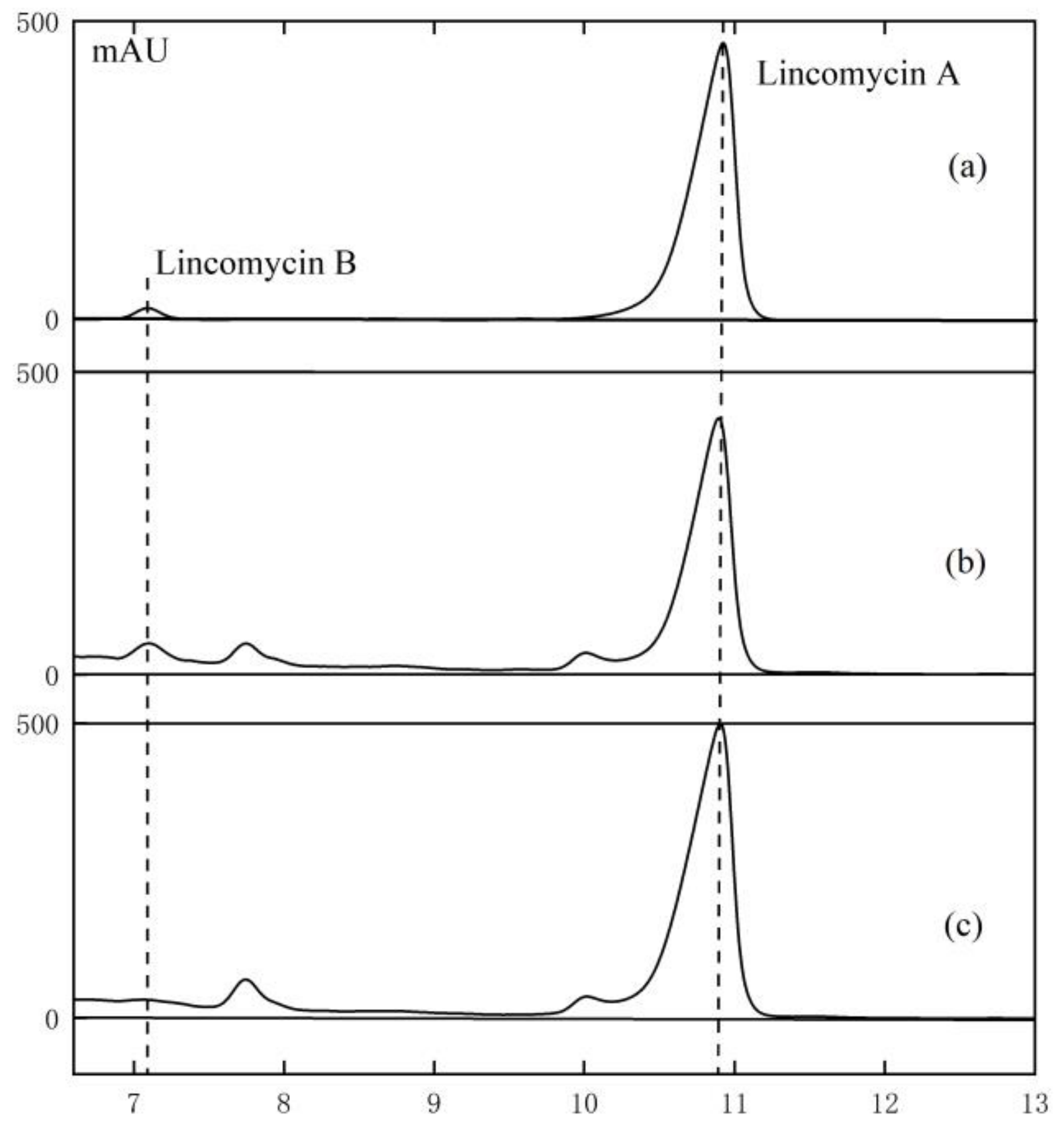

Figure 2

HPLC analysis of lincomycin production during Streptomyces. lincolnensis fermentation. (a) Standard lincomycin A and lincomycin B. (b) Lincomycin A and lincomycin B produced by the initial medium. (c) Lincomycin $A$ and lincomycin $B$ produced by the optimized medium. 

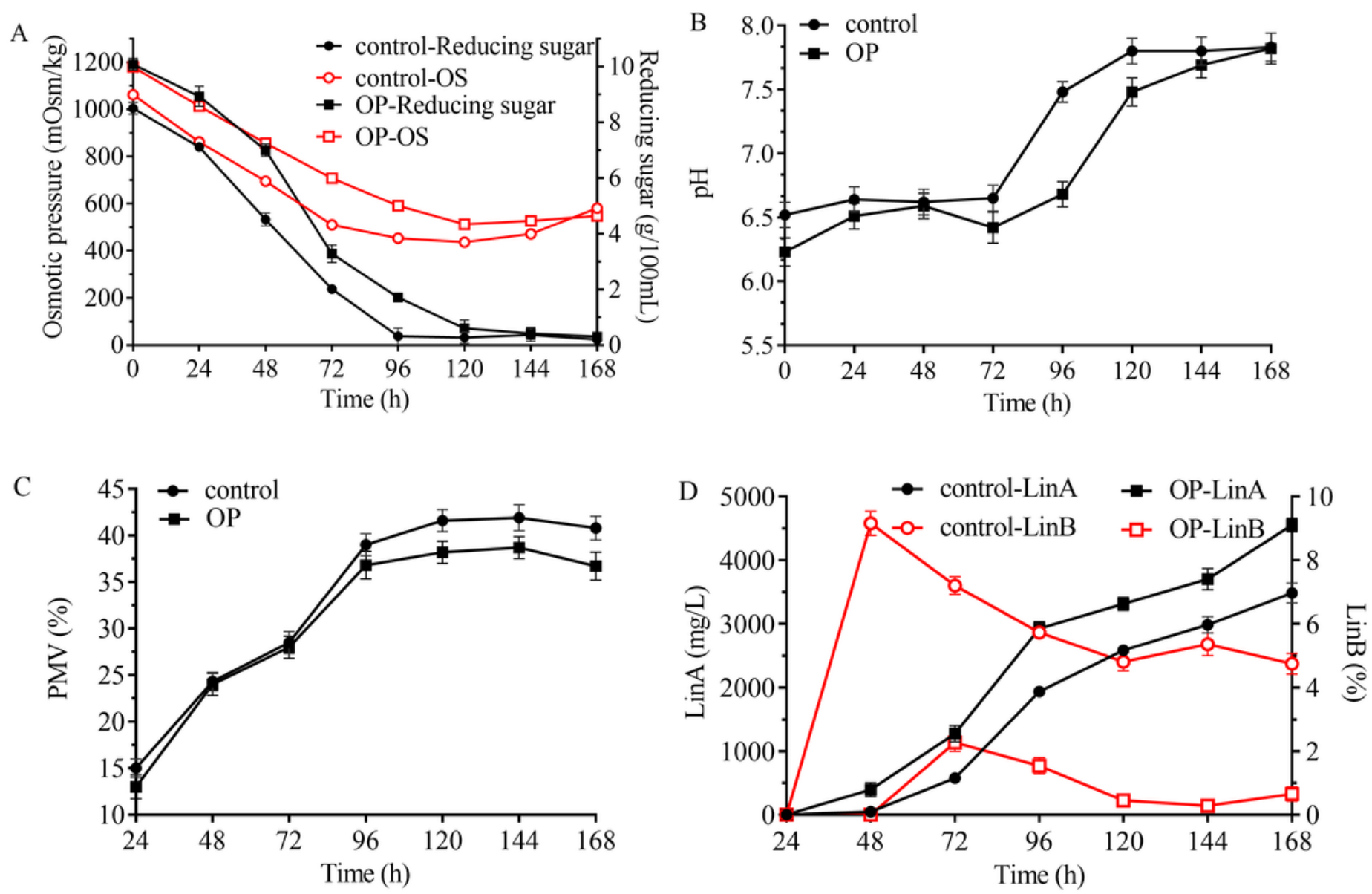

Figure 3

Fermentation time-course profile of Streptomyces lincolnensis grown in the optimized and the initial media. (A) Reducing sugar concentration and osmotic stress level during fermentation. (B) pH evolution; (C) PMV evolution; and (D) production of lincomycin A and lincomycin B in initial and optimized media. Control: initial medium; OP: optimized medium; OS: osmotic stress. 


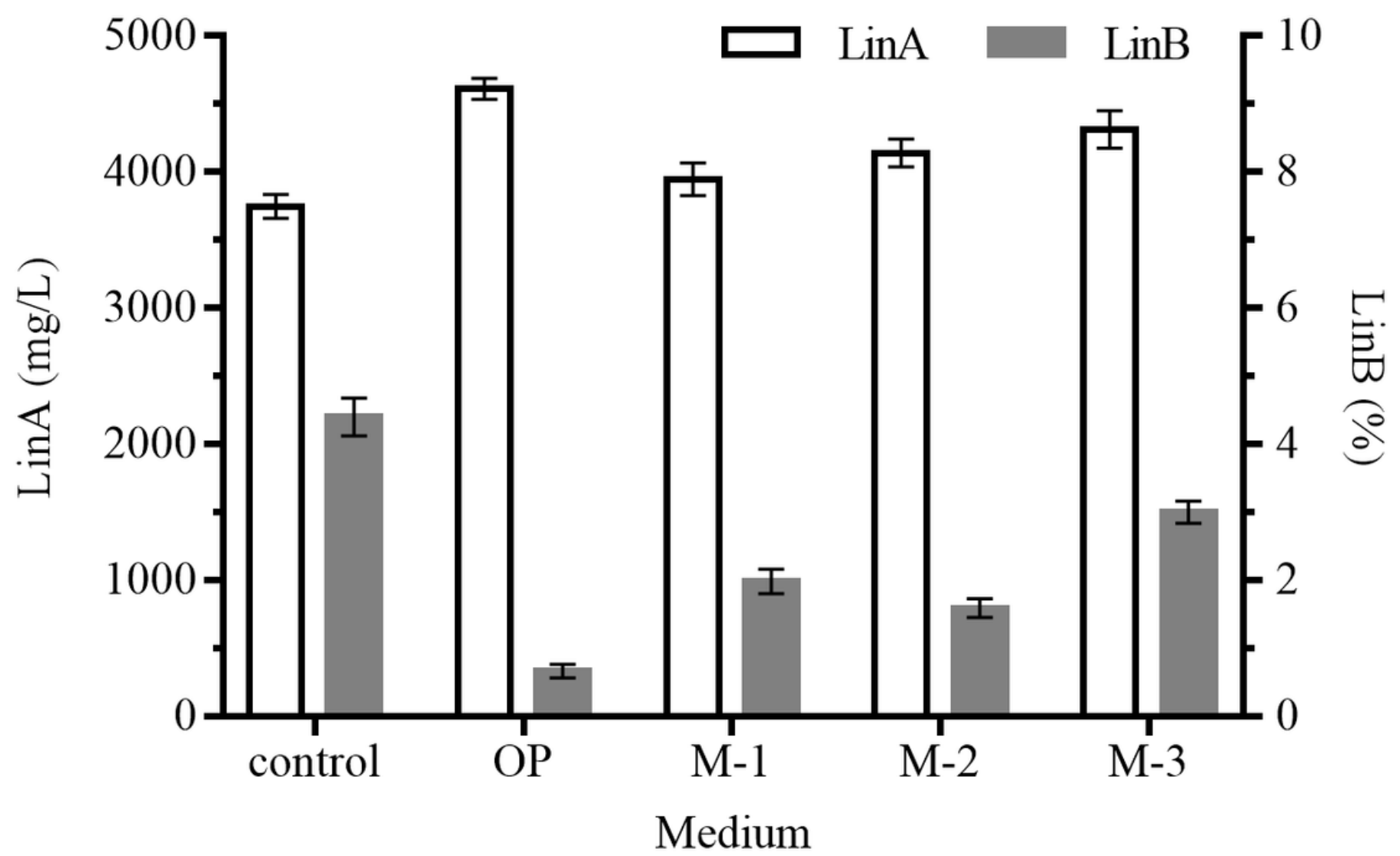

Figure 4

Effects of different media on lincomycin A yield and lincomycin B concentration. Control: initial medium. The experimental media included the same components as the initial medium except for the following differences: OP: optimized medium and $1.4 \mathrm{~g} / \mathrm{L}$ corn steep liquor and $126 \mathrm{~g} / \mathrm{L}$ glucose; M-1: $10 \mathrm{~g} / \mathrm{L} \mathrm{NaCl}$; M-2: $126 \mathrm{~g} / \mathrm{L}$ glucose; and M-3: $1.4 \mathrm{~g} / \mathrm{L}$ corn steep liquor. 


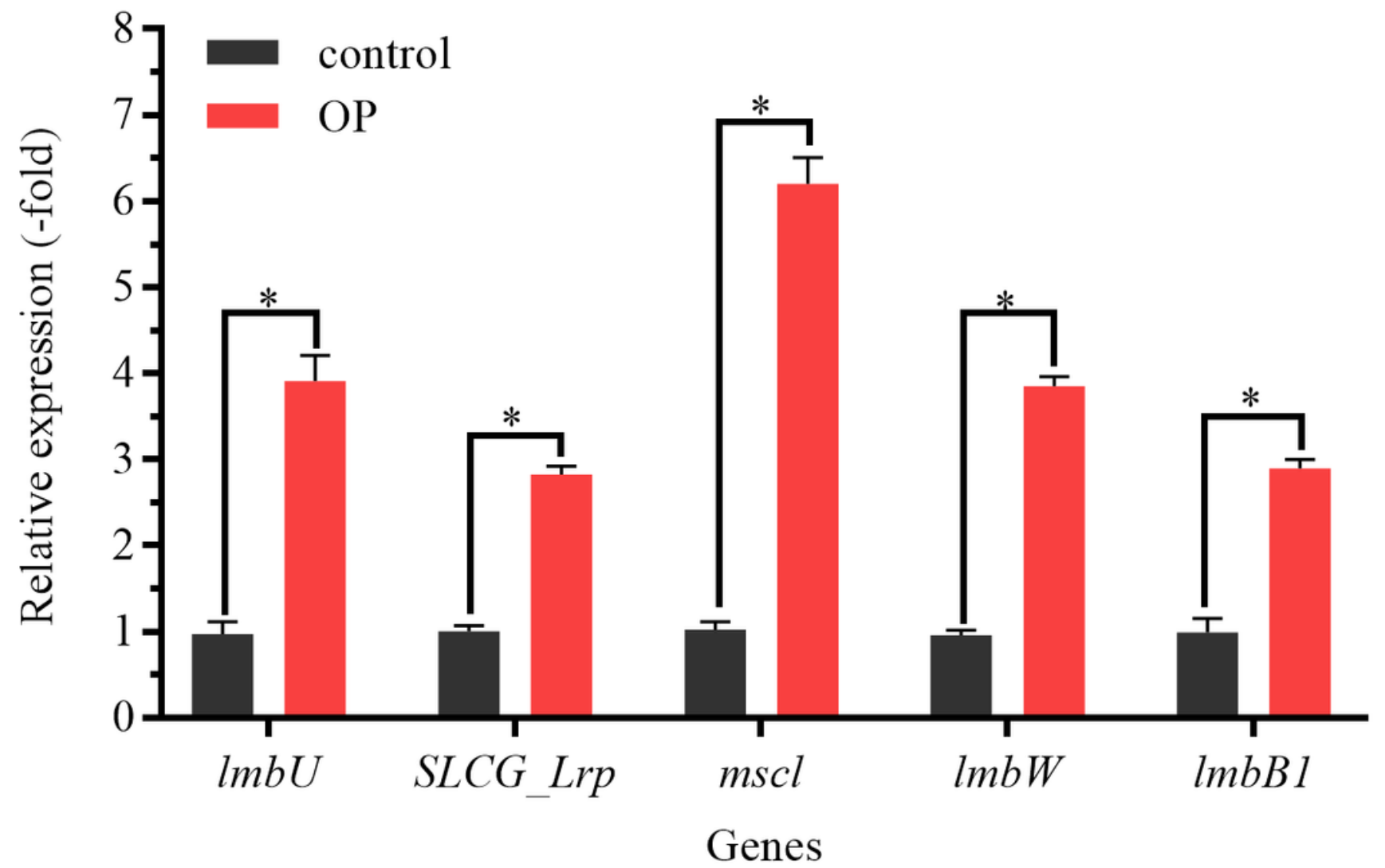

Figure 5

Transcriptional analysis of the lincomycin biosynthetic genes and osmotic stress gene in Streptomyces lincolnensis grown in initial and optimized media. All gene transcription levels in the control were set to 1 . * indicates statistical significance based on the two-tailed test $(p<0.05)$. Control: initial medium; OP: optimized medium.

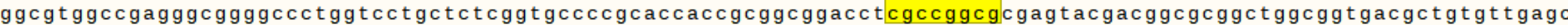

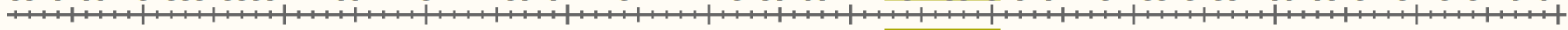

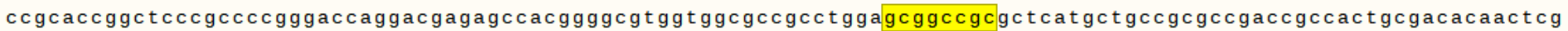

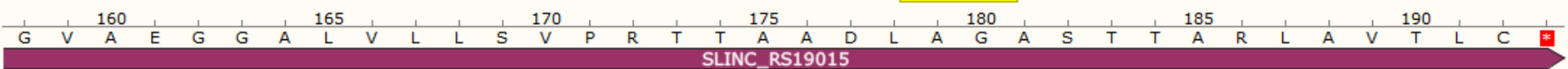

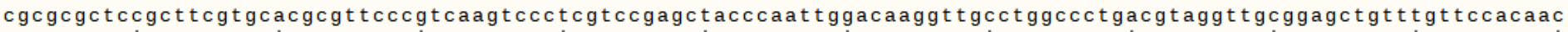

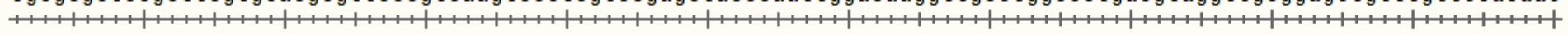

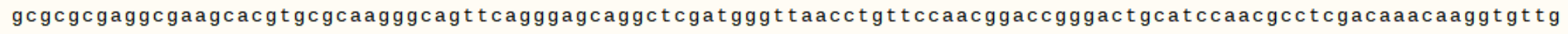

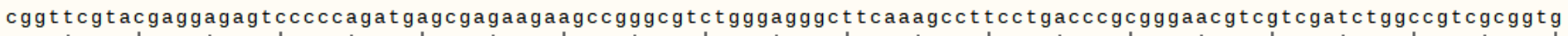

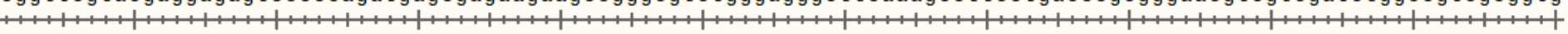

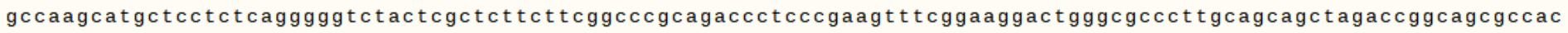

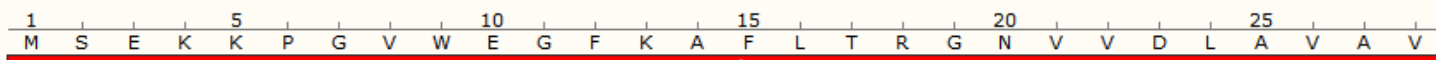

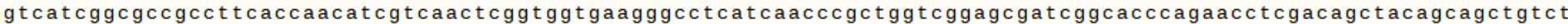

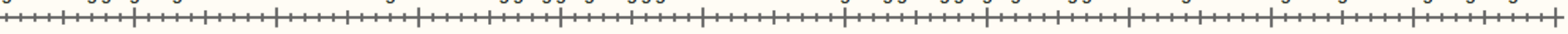

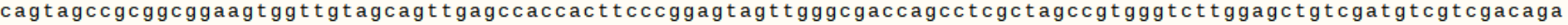

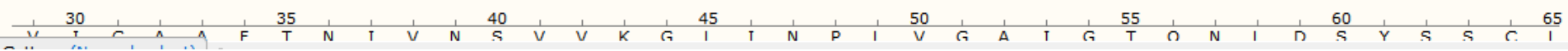


Figure 6

Nucleotide sequence of the region upstream of mscl and the predicted LmbU binding site (yellow).

\section{Supplementary Files}

This is a list of supplementary files associated with this preprint. Click to download.

- Supportinginformation.doc 